\title{
Candida albicans infection among HIV positive and HIV negative women- Case study at Butare University Teaching Hospital (CHUB), Southern province of Rwanda
}

\author{
Ella Larissa Ndoricyimpaye ${ }^{\mathrm{a}^{*}}$, Tuyishime Obed ${ }^{\mathrm{a}}$, Habiyakare Jean Claude ${ }^{\mathrm{a}}$, Manishimwe Jean \\ d'Amour ${ }^{\mathrm{a}}$, Ntwali Denyse ${ }^{\mathrm{a}}$, Rutayisire Reverien ${ }^{\mathrm{a}}$. \\ aUniversity of Rwanda, College of Medicine and Health Sciences, Department of Biomedical Laboratory Sciences, \\ Kigali- Rwanda \\ *Correspondence to Ella Larissa Ndoricyimpaye (ellalarissan@gmail.com)
}

\begin{abstract}
Background: Candida albicans still major leading global health challenge especially in immunocompromised individual particularly living in low-income countries with limited access to the antifungal drugs. Though the incidences and prevalence of opportunistic infection have been reduced due to the availability and strict adherence to the antiretroviral therapy, candidiasis remains the most frequent fungal infection with high morbidity and mortality and It is still a neglected topic in the research sectors of developing world including Rwanda. This study aimed to determine the prevalence of candida albicans infection among HIV positive and HIV negative women attending Butare University Teaching Hospital in the southern province of Rwanda.

Methods: A descriptive cross-sectional study was conducted with 306 female participants aged between 17-64 years old. Conventional sampling method was used to obtain the desired population and an interviewer structured questionnaire was administered to gather social demographic information and associated risk factors. both urine and a drop of blood for HIV were requested for testing. HIV testing followed the national HIV testing policy.

Results: Among 306 women participated; the average age range was 30-40 ages. The overall prevalence of candida albicans was $10.5 \%$ and was highly isolated in HIV positive women 21(13.7\%) than in HIV negative women 11(7. 2\%). Pregnancy and antibiotics usage were statistically significant associated with candidiasis with p-value $<0.05$ while age was not statistically associated with candida albicans infection with p-value $>0.05$.

Conclusion: The availability and strict adherence to antiretroviral therapy to people living with HIV have tackled the opportunistic infections like candidiasis. However, poor hygiene still the major health challenge for both groups. Hence, improved hygiene and early treatment with an improved diagnostic test to the risk population should highly be importance.
\end{abstract}

Key words: Prevalence, Candida albicans infection, Butare University Teaching Hospital.

\section{INTRODUCTION}

T ungal infections continue to pose significant morbidity 1 and mortality due to the increasing number of at-risk population across the world particularly in developing countries $^{1,2}$. Globally, an estimated 37.9 million people were living with HIV in $2018^{3}$. HIV/AIDS officially recognized as epidemic since the 1980 s with the increase of immunocompromised individual that has exposed them to numerous opportunistic infections including candida

East Africa Science 2020| Volume 2 | Number 1 infection of fungal aetiology and accompanied with massive population death ${ }^{4,5}$. Candida species including C. albicans, C. tropicalis, C. glabrata, C. kruzei, C. dublinensis, $C$. guilliermondii, C. parapsilosis, C. kefyr, and C. pelliculosa are the most frequent fungal species of the human microbiota and these species asymptomatically colonizes many areas of the body, predominantly the gastro-intestinal and genitalurinary tracts of healthy individuals ${ }^{6,7}$. However, in the condition that could impair the host immune system, C. 
albicans can change from normal commensal state to a pathogenic state by penetrating the mucosal surface and cause a deep-seated invasive and life-threatening infection ${ }^{8}$. The pathogenic state of $C$. albicans is highly associated with its ability to undergo phenotyping switching from different cellular form like yeast cell to hyphae cell depending on environment growth conditions $\mathrm{s}^{9,10,11}$.

C. albicans remains the most frequent pathogenic candida species responsible for almost fungal morbidity and mortality in the developing world and causing the increased cost of care and duration of hospitalization ${ }^{12}$. The nonalbicans candida species have significantly emerged to cause infection in patients living with HIV due to the fact that these non-albicans species are less susceptible to the commonly used antifungal drugs which hinder their effective treatment ${ }^{13}$. Although $C$. albicans remains the dominant species causing Blood Stream Infection, the frequency of occurrence varies throughout the world from a low of $37 \%$ in Latin America to a high of $70 \%$ in Norway ${ }^{14}$. The recent global incidence was estimated to be 700,000 cases of invasive candidiasis and reported to have a mortality rate of $38 \%$ to $44 \%$ in developing countries with resource-limited settings where conventional diagnostics test is slow, expensive or delay to access the antifungal drugs in all countries and a high burden of HIV coinfection ${ }^{15}$.

In Africa and the Middle East, Candida albicans is highly isolated to cause invasive and superficial candidiasis with the prevalence of $55.18 \%$ and $76.92 \%$ respectively. ${ }^{16}$ This was mostly seen in Sub-Saharan Africa and central Africa where Candida albicans infections rate was (22.74\%), followed by the South African region (22.44\%),. With regard to $C$. albicans invasive infections still prevalent in Sub Saharan and central Africa at (12.34\%), followed by the Middle East $(6.05 \%)^{16}$. Vaginal C. albicans infection has been implicated most often in a number of candida infections which affects approximately two-thirds of the women of reproductive age globally. Candida may become worse in a patient with the compromised immune system, such as HIV/AIDS patients or those that are on immunosuppressive therapies, broad-spectrum antibiotics users and pregnant women, this is a potentially life-threatening leading to systemic fungal infection ${ }^{17}$.

In Rwanda, Candida infection and other fungal aetiology disease are still under-diagnosed with routine diagnostic tests and rarely considered as a global health challenge particularly in immunocompromised individuals that could result in drug resistance. Moreover, there is limited data with no published information mainly in research sectors oriented on candidiasis highlighting the burden of the aforementioned infection in HIV positive or HIV negative individuals that could contribute to the ineffectiveness of treatment if not properly managed. Thus, this study is aimed to determine the prevalence of Candida albicans infection among HIV positive and HIV negative women attending Butare University Teaching Hospital in the southern province of Rwanda.

\section{METHODS}

\section{Design and Study area}

This was a hospital-based descriptive cross-sectional study conducted from May to September 2019 at Butare University Teaching Hospital. It is located at Mamba, Butare Cell, Huye District in the Southern province of Rwanda, a National Referral Hospital which serves more than 3,772,230 people from Southern Province and others from different locations of Rwanda.

\section{Sample size determination \& Sampling}

A convenience sampling strategy was used for participant's selection. By referring to the prevalence of Candida albicans $27.5 \%$ in the previous study conducted in Uganda ${ }^{18}$. The minimum sample size was calculated using Fisher's formula

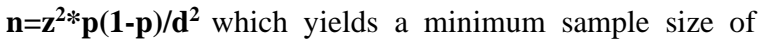
$306^{19}$. Where $\mathbf{n}=$ sample size, $\mathbf{z}=\mathbf{z}$ statistic for the level of confidence i.e.1.96, $\mathbf{P}=$ expected prevalence $(27.5 \%)$ and $\mathbf{d}=$ allowable error i.e. 0.05 .

\section{Data collection and processing}

Participants meeting the inclusion criteria and willing to participate in the study were conveniently recruited from the ART (Antiretroviral Therapy) clinic and OPD (Outpatients Department) service. The study was mainly intended to isolate the pathogenic microorganism, therefore those who were using the antifungal treatments were excluded from the study.

The informed consent forms were given together with a semi-structured questionnaire to gather demographic information. The participants from the ART clinic were given urine containers and requested to bring urine sample whereas those from OPD department were requested to give blood for HIV status testing as an addition to urine samples. The HIV status testing followed the national HIV testing policy by use of Alere ${ }^{\circledR}$ HIV combo for screening and Stat Pak for confirmation. The participants confirmed positive HIV test result was counselled by Hospital counsellors of the ART clinic. All 306 urine samples were streaked over Sabouraud Dextrose Agar media -SDA and incubated aerobically at $37^{\circ} \mathrm{C}$ for 3-4 days. Yeast growths were then identified by colonial morphology characteristic of Candida on above-mentioned media. For confirmation of Candida albicans using germ tube test, a single colony from SDA was 
Candida albicans infection among women at Butare University Teaching Hospital (CHUB),

isolated, transferred and emulsified in human serum for 4 hours at $37^{\circ} \mathrm{C}$.

\section{Data analysis method}

The data were entered into Microsoft Excel (Microsoft Corp, Santa Rosa, CA, USA) and exported for statistical analysis in IBM SPSS Statistical developer version 21. Relative frequency of demographic characteristics was summarized using descriptive statistic. Correlation of dependent and independent variables was analyzed using Chi-square test. The level of statistical significance was set at $95 \%$ confidence level where $\mathrm{p}$-value $<0.05$.

\section{Ethical considerations}

Ethical approval was obtained from the Institution Review Board, University of Rwanda/ College of Medicine and Health Sciences and Ethical Committee of Butare University Teaching Hospital with reference numbers CMHS/IRB/219/2019 and RC/UTHB/057/2019 respectively. The participants were explained about the study, only participants with signed informed consent form were enrolled in the study. Moreover, Ethical clearance was distributed to the head of the respective department.

\section{RESULTS}

The study included 306 women participants of the average age range of 31-40 years old of which 153 were HIV positive and 153 were HIV negative women from ART clinic and OPD department respectively (table1). Candida species were isolated in 59(19.2\%) out of total population which account $32(54.2 \%)$ of germ tube formers isolated as Candida albicans and 27(45.7\%) germ tube test negative isolated as Candida non-albicans (data not shown). C. albicans was highly isolated among ART participants 21(13.7\%) compared to OPD participants 11(7.2\%) (Table 1). The overall prevalence of $C$. albicans was $10.5 \%$ while Candida non-albicans accounted for $8.8 \%$ (Table 1). However, Candida albicans was significantly associated with antibiotics usage and pregnancy status in both HIV positive and HIV negative women with p-value $<0.05$ (Table 2).

TABLE 1. Demographic characteristic of study population in relation to Candida species

\begin{tabular}{|c|c|c|c|c|c|}
\hline \multirow[b]{2}{*}{ Variables } & \multirow[b]{2}{*}{$\mathrm{n}$} & \multicolumn{2}{|c|}{ Microorganisms isolated } & \multirow[b]{2}{*}{ Negative $(\%)$} & \multirow[b]{2}{*}{$p$-value } \\
\hline & & C. albicans (\%) & C. non albicans (\%) & & \\
\hline \multicolumn{6}{|l|}{ Age, years } \\
\hline$<20]$ & 7 & $0(0 \%)$ & $1(14.3 \%)$ & $6(85.7 \%)$ & \\
\hline [21-30] & 87 & $16(18.4 \%)$ & $8(9.2 \%)$ & $63(72.4 \%)$ & \\
\hline [31-40] & 123 & $7(5.7 \%)$ & $14(11.4 \%)$ & $102(82.9 \%)$ & 0.084 \\
\hline [41-50] & 55 & $7(12.7 \%)$ & $2(3.6 \%)$ & $46(83.6 \%)$ & \\
\hline$>50$ & 34 & $2(5.9 \%)$ & $2(5.9 \%)$ & $30(88.2 \%)$ & \\
\hline \multicolumn{6}{|l|}{ HIV status } \\
\hline Positive & 153 & $21(13.7 \%)$ & $8(5.2 \%)$ & $124(81 \%)$ & 0.002 \\
\hline Negative & 153 & $11(7.2 \%)$ & $19(12.4 \%)$ & $123(80.4 \%)$ & \\
\hline Total & 306 & $32(10.5 \%)$ & $27(8.8 \%)$ & $247(80.7 \%)$ & \\
\hline
\end{tabular}

TABLE 2. Distribution of Candida albicans among study population associated with pregnancy and antibiotics usage status

\begin{tabular}{llcccc}
\hline & Variables & & Participants n & C. albicans (\%) & P-value \\
\hline HIV & Pregnant & Yes & 5 & $3(60 \%)$ & 0.002 \\
Positive & & No & 148 & $18(12.2 \%)$ & \\
& & 19 & $7(36.8 \%)$ & 0.002 \\
& Antibiotics Usage & Yes & 134 & $14(10.4 \%)$ & \\
& & No & &
\end{tabular}


Candida albicans infection among women at Butare University Teaching Hospital (CHUB), Southern province of Rwanda

www.eahealth.org

\begin{tabular}{llcccc}
\hline HIV & Pregnant & Yes & 29 & $8(27.6 \%)$ & 0.001 \\
Negative & & No & 124 & $3(2.4 \%)$ & \\
& & & 20 & $5(25 \%)$ & 0.001 \\
& Antibiotics Usage & Yes & 133 & $6(4.5 \%)$ & \\
\hline & & No & & \\
\hline
\end{tabular}

\section{DISCUSSION}

Candida albicans infection remains a major public health concern particularly among immunocompromised individuals in developing countries with limited resources including Rwanda. Understanding its prevalence and associated risk factors can be a major contribution to the effective control and preventive measures. The present study revealed $10.5 \%$ as the prevalence rate of Candida albicans in the entire study population and was the most isolated species considered with other Candida species. This is in accordance with the works reported elsewhere in a different study which stated that $C$. albicans is the most frequent colonizer and responsible for almost clinical manifestation of candidiasis ${ }^{20,21}$.

The prevalence of $10.5 \%$ in this study is low compared to $22.3 \%$ and $32.4 \%$ that reported among women in Kigali University Teaching Hospital, Rwanda and Lagos Teaching Hospital, Nigeria $^{20,22}$. This reduction of prevalence could be attributed to the fact of availability of antiretrovirals to the patients, strict adherence to their antiretroviral regimen and sensitive sampling method (vaginal swabs) used.

Considering the prevalence among the study populations; $C$. albicans in HIV positive women was high $13.7 \%$ compared to $12.5 \%$ and $9.7 \%$ of the seropositive population in Nigeria $^{23,24}$. This could be due to the fact that they included both HIV positive male and female who were on antiretroviral therapy with the exclusion of the associated risk factors. Contrary, it was low compared to $22.3 \%$ and $50 \%$ reported among seropositive patients in Kigali and India respectively ${ }^{13,22}$.

As observed in the present study, the prevalence of Candida albicans among the study population in relation to pregnant status was similar to $34.3 \%$ reported among pregnant women in a federal medical centre, Nigeria ${ }^{20}$. This was supported by the facts that, pregnancy could be a risk factor which increases the possibility of getting candida infections due to the increases of the sensitivity of vagina to infection and increase of reproductive hormones that elevate the amount of glycogen in the vagina which can suppress the normal vaginal environment ${ }^{20,25}$. The 21-30 age groups were shown to be more susceptible to candida infection compare to other groups (Table 1). This work agreed with that reported in the previous studies which showed that 20-29 age groups had a higher prevalence of candida colonizers with predominant aetiology of $C$. albicans to cause vaginal candidiasis ${ }^{26}$. This could be attributed to the fact of the active age group with the highest risk of pregnancies, indulgence in family planning pills and immunosuppression due to HIV/AIDS. Contrary, the population aged 46 years or above have reached menopause and have shown to develop resistance to candida infection due to the fact that, they are less or not sexually active, not using contraceptive drugs to prevent pregnancy and they have increased vaginal immunity as they have reduced the level of estrogen and corticoid ${ }^{21}$.

With the regards to the antibiotics usage facts, the prevalence of $C$. albicans among antibiotics users living with HIV was $36.8 \%$ and $10.4 \%$ for antibiotics non-users while the prevalence of C. albicans among antibiotics users and nonusers who were HIV negative was $25 \%$ and $4.6 \%$ respectively. These results disagreed with that of reported among women in primary health care centres of Jos, Nigeria that, the broad-spectrum antibiotics users accounted for $16 \%$ and $33.6 \%$ respectively as the risk for vaginal candidiasis $^{20,26}$. The fact of using antibiotics can be a predisposing factor that leads to candida colonization but the absence of this factor does not necessarily defend against Candida colonization ${ }^{25}$.

\section{CONCLUSION}

Low prevalence of Candida albicans infection was significantly observed compared to that conducted in Rwanda in 1995. Pregnancy and antibiotics usage was revealed to significantly contribute to candida infection. Thus, regular and early medical checkup and a regular public education on the importance of personal hygiene and proper use of antibiotic should reduce disease burden. Further research should be conducted to explore more about $C$. albicans infection and burden of the fungal etiological agent in women by using different sampling method and improved diagnostic test.

\section{Limitation}

This study was limited by small sample size and less sensitive samples used which might have interfered with the accuracy of the results. 
Acknowledgement: Our Sincere gratitude is addressed to the department of Biomedical Laboratory Sciences for incomparable pieces of advice and effort during the implementation of this project. Moreover, we thank the staff of Huye Biotechnology Complex Laboratory for their support of materials \& place during data collection and Directorate of CHUB for their help in the recruitments of participants. Heartfelt gratitude goes to all women who accepted to participate in the study.

\section{REFERENCE}

1. Bongomin F. Global and Multi-National Prevalence of Fungal Diseases — Estimate Precision. J Fungi. 2017;57(3):29. DOI: $10.3390 /$ jof3040057

2. Guto JA, Bii CC, Denning DW. Emerging Problems in Infectious Diseases- the Estimated burden of fungal infections in Kenya. $J$ Infect Dev Ctries. 2016;10(8):777-784. DOI:10.3855/jidc.7614

3. WHO. World Health Statistics - Monitoring Health for the SDGs. Geneva: World Health Organization; 2018.

4. Nobile CJ, Johnson AD. Candida albicans Biofilms and Human Disease. Annu Rev Microbiol. 2015;69:71-92. DOI:10.1146/annurevmicro-091014-104330

5. Nancy K, Anne O brien. e. al. Overview of HIV. Psychosom Med 2008;70:523-530. DOI:10.1097/PSY.0b013e31817ae69f

6. Sardi JC., L. S, T B, A. M F-A, M. J. S MG. Candida species : current epidemiology, pathogenicity, biofilm formation, natural antifungal products and new therapeutic options. J Med Microbiol. 2013;64:10 24. DOI: $10.1099 /$ jmm.0.045054-0

7. Darwazeh AMG, Darwazeh TA. What Makes Oral Candidiasis Recurrent Infection? A Clinical View. J Mycol. 2014;14:1-5. DOI: $10.1155 / 2014 / 758394$

8. Nikou S, Kichik N, Brown R, et al. Candida albicans Interactions with Mucosal Surfaces during Health and Disease. Pathogens. 2019;8(53):1-23. DOI:10.3390/pathogens 8020053

9. Arkowitz RA, Bassilana M. Recent advances in understanding Candida albicans hyphal growth [ version 1 ; peer review : 4 approved ]. F1000Research 2019;8(700):1-10. DOI:10.12688/f1000research.18546.1

10. Carolus H, Dyck K Van, Dijck P Van. Candida albicans and Staphylococcus Species: A Threatening Twosome. Front Microbiol. 2019;10(2162):1-8. DOI:10.3389/fmicb.2019.02162

11. Repentigny L De, Lewandowski D, Jolicoeur P. Immunopathogenesis of Oropharyngeal Candidiasis in Human Immunodeficiency Virus Infection. Clin Microbiol Rev. 2004;17(4):729-759. DOI:10.1128/CMR.17.4.729

12. Morgan J, Meltzer MI, Plikaytis BD, Andre NS, White SH, Wilcox S. Excess mortality, hospital stay and cost due to Candidemia : a casecontrol study using data from Population-based Candidemia Surveillance. Infect Control Hosp Epidemiol. 2005;26(6):540-547. DOI: $10.1086 / 502581$

13. Maheshwari M, Kaur R, Chadha S. Candida Species Prevalence Profile in HIV Seropositive Patients from a Major Tertiary Care Hospital in New Delhi, India. J Pathog. 2016;2016:8. DOI: $10.1155 / 2016 / 6204804$

14. Pfaller MA, Diekema DJ, Isolates C, Ic F. Epidemiology of Invasive Candidiasis: a Persistent Public Health Problem. Clin Microbiol Rev. 2007;20(1):133-163. DOI:10.1128/CMR.00029-06

15. Wilma C, Africa J, Miguel P. Candida antifungal drug resistance in
sub-Saharan African populations : A systematic review [ version 2 ; referees : 2 approved ] Referee Status : F1000Research. 2017;5(2832):1-9. DOI:10.12688/f1000research.10327.1

16. Omrani AS, Pecen L, Hajek P, Raghubir N, Zigmond J. Prevalence of invasive and superficial Candida infections in Africa and the Middle East ; a Systematic Review and Meta-analysis. Cent East Eur Outcomes Res. 2014;48(5):17-20.

17. Mnge P, Okeleye BI, Vasaikar SD, Apalata T. Species distribution and antifungal susceptibility patterns of Candida isolates from a public tertiary teaching hospital in the Eastern Cape Province, South Africa. Brazilian J Med Biol Res. 2017;50(6):1-7. DOI:10.1590/1414431X20175797

18. Ocan M, Tumushabe B, Nakawunde H, et al. Prevalence and Antifungal Susceptibility of Vaginal Candida albicans among Pregnant Women Attending Arua Regional Referral Hospital, West Nile Region of Uganda. Acta Sci Microbiol. 2018;1(6):17-22.

19. Arya R, Antonisamy B. Sample Size Estimation in Prevalence Studies. Indian J Pediatr. 2012;79(11):1482-1488. DOI:10.1007/s12098-012-0763-3

20. Uzoh C., Iheukwumere I., Umezurike K., Onyewenjo S. Prevalence of Candida albicans among Women Attending Federal. Adv Life Sci Technol. 2016;41:54-58.

21. Emeribe AU, Idris Abdullahi N, Justus O, Alinwachukwu Loveth I Prevalence of vulvovaginal candidiasis among nonpregnant women attending a tertiary health care facility in Abuja, Nigeria. Res Rep Trop Med. 2015;6:37-42. DOI:10.2147/RRTM.S82984

22. Leroy V, Clercq A De, Ladner J, Bogaerts J, Perre P Van De. Should screening of genital infections be part of antenatal care in areas of high HIV prevalence? A prospective cohort study from Kigali, Rwanda. Genitourin Med. 1995;71(8):207-211. DOI:10.1136/sti.71.4.207

23. Okonkwo EC, Alo MN, Nworie O, Orji JO, Agah M V. Prevalence of oral candida albicans infection in HIV seropositive patients in Abakaliki. Am J Life Sci. 2013;1(2):72-76. DOI:10.11648/j.ajls.20130102.18

24. Lar PM, Pam K V, Yop T, et al. Prevalence and distribution of Candida Species in HIV infected persons on antiretroviral therapy in Jos. J Med Med Science. 2012;3(4):254-259.

25. Jack.D.Sobel. Current concepts: Vaginitis. $N$ Engl J Med. 1998;337(12):1-8

26. Nwadioha SI, Egah DZ, Alao OO, Iheanacho E. Risk factors for vaginal candidiasis among women attending primary health care centres of Jos, Nigeria. J Clin Med Res. 2010;2(7):110-113.

Peer Reviewed

Competing Interests: None declared.

Received: 18 Feb 2019; Accepted: 07 May 2020.

Cite this article as: Ndoricyimpaye EL, Obed T, Claude HJ, d'Amour MJ, Denyse N, Reverien R. Candida albicans infection among HIV positive and HIV negative women- Case study at Butare University Teaching Hospital (CHUB), Southern province of Rwanda. E Afr Sci. 2020;1(2):75-79. http://doi.org/10.24248/EASci-D-19-00003

(C) Ndoricyimpaye et al. This is an open-access article distributed under the terms of the Creative Commons Attribution License, which permits unrestricted use, distribution, and reproduction in any medium, provided the original author and source are proper-ly cited. To view a copy of the license, visit http://creativecommons.org/licens- es/by/4.0/. When linking to this article, please use the following permanent link: http://doi.org/10.24248/EASci-D-19-00003 